\title{
Expression of platelet derived growth factor and platelet derived growth factor receptor mRNA in a glioblastoma from a patient with Li-Fraumeni syndrome
}

\author{
Abhijit Guha, Danuta Glowacka, Rona Carroll, Kathleen Dashner, Peter McL Black, \\ Charles D Stiles
}

\begin{abstract}
Expression of platelet derived growth factor (PDGF) and PDGF-receptor mRNA was examined from a glioblastoma taken from a patient with LiFraumeni syndrome. Northern blot analysis and in situ hybridisation showed very high concentrations of both PDGF$A$ and PDGF $a$-receptor mRNA in the tumour. The overall pattern of PDGF expression was similar to those found in sporadic glioblastomas. Mutations in p53 has been implicated as an early pathogenic event leading to sporadic low grade astrocytomas, and is the third most common tumour type in patients with $\mathrm{Li}$ Fraumeni syndrome, where they are predisposed due to a germline mutation in the p53 tumour suppressor gene. This study suggests that progression towards a glioblastoma in both the general population and in patients with Li-Fraumeni syndrome may involve potential autocrine and paracrine stimulation by growth factors such as PDGF.
\end{abstract}

(F Neurol Neurosurg Psychiatry 1995;58:711-714)

Keywords: astrocytoma; platelet derived growth factor; Li-Fraumeni syndrome

Dana-Farber Cancer Institute, Boston, USA A Guha

C D Stiles

Division of

Neurosurgery and

Surgical Oncology,

University of Toronto,

Toronto, Canada

A Guha

Division of

Neurosurgery,

Brigham and Womens

Hospital, Boston, USA

D Glowacka

R Carroll

K Dashner

P McL Black

Correspondence to:

Dr Abhijit Guha, 2-415

McLaughlin Pav Toronto

Hospital, Western Division

399 Bathurst St, Toronto,

Ontario, Canada M5T-2S8.

Received 23 September 1994

and in revised form

3 January 1995

Accepted 10 January 1995

Patients with $\mathrm{Li}$-Fraumeni syndrome harbour a germline mutation in the p53 tumour suppressor gene, and have a $50 \%$ chance of developing at least one malignancy by age $30 .^{1}$ Astrocytomas, occurring at a younger age than in the general population, are the third most common of the six cancer types presently associated with the syndrome. Malignant astrocytomas from patients with Li-Fraumeni syndrome and those from the general population are pathologically indistinguishable.

Both PDGF and PDGF receptors are expressed by most malignant astrocytomas cell cultures, and their tumorogenic growth can be reversed by blocking PDGF autocrine and paracrine stimulation. ${ }^{2}$ Recently we have identified increased concentrations of PDGF and PDGF receptors at both the mRNA and protein level in sporadically occurring glioblastoma specimens taken at operation. ${ }^{3}$ Whether PDGF is also important in the development of glioblastomas where there is a cancer predisposing germline defect, such as in the Li-Fraumeni syndrome, is not known and is the subject of this study.

\section{Case and family history}

A 26 year old man presented with a three month history of headaches. Brain CT showed a left frontal enhancing mass, which was subtotally resected and diagnosed as a glioblastoma (specimen OR1). Five months after external radiation, progressive symptomatic enlargement of the residual tumour necessitated a second debulking (specimen OR2). Lesions were noted in both hemispheres by 18 months, and were confirmed by biopsy to be multifocal glioblastomas. Members of four successive generations in the patient's family had succumbed to malignancies at an early age, in a autosomal dominant pattern of cancer transmission with high penetrance (fig 1). On this clinical-epidemiological basis, a diagnosis of Li-Fraumeni syndrome was made. Our patient was the first member with a verified CNS tumour in this family.

\section{Methods}

TISSUE COLLECTION

Specimens for northern analysis (OR1 OR2) were snap frozen. For in situ hybridisation, the specimens (OR2 only) were immediately immersed in freshly prepared $4 \%$ 
Figure 2 Northern blot analysis for PDGF-A and $B(A)$ and PDGF $\alpha$ and $\beta$-receptor (B) $m R N A$. Two specimens of glioblastoma from the

patient with Li-Fraumeni syndrome (OR1 and

$O R 2)$ and cell lines serving as positive controls [U343 (glioblastoma) for PDGF$A$ and $B ; U 373$

(glioblastoma) for PDGF

$a$-receptor and SK76

(human fibroblast; FIBR)

for PDGF $\beta$-receptor] were

examined. A specimen

labelled BRAIN, from a

patient diagnosed with

mesial temporal sclerosis

was used to represent non-

neoplastic brain. Roughly

equal amounts of $R N A$

were transferred on the

blots as seen by the

corresponding ethidium

bromide stained $28 S$

ribosomal $R N A$ bands.

$P D G F \alpha$-receptor $m R N A$

was greatly increased in

the glioblastomas,

especially in the OR1

specimen, whereas PDGF

$\beta$-receptor mRNA was

barely detected. PDGF- $A$

$(2 \cdot 8,2 \cdot 2$, and $1.6 \mathrm{~kb})$

and to a lesser degree

$P D G F-B$ mRNA were

both increased in the

glioblastoma specimens.
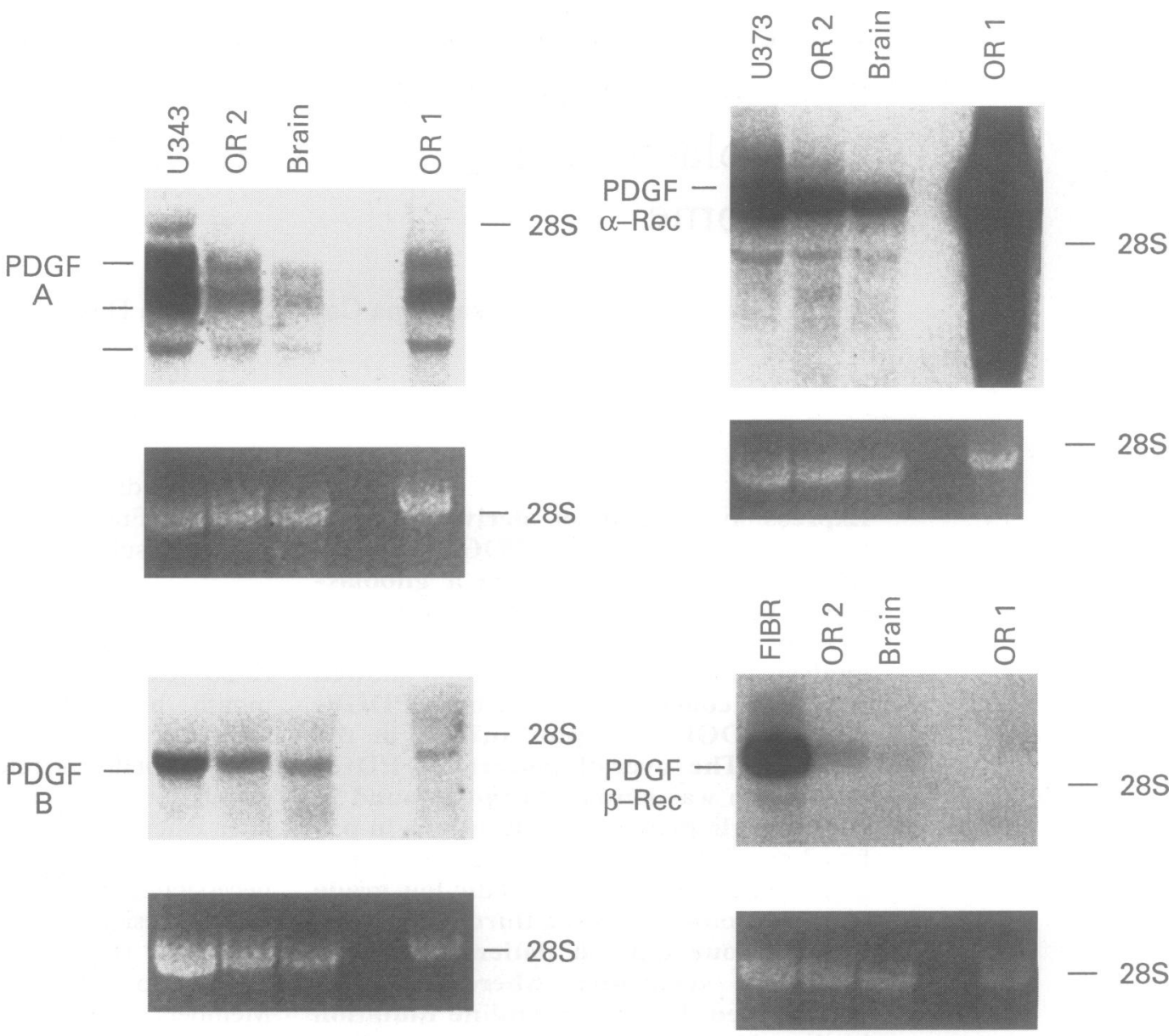

A

B

paraformaldehyde/phosphate buffered saline (PBS) solution, and then immersed in a $30 \%$ sucrose/PBS buffer overnight. Cryosections of $6 \mu \mathrm{m}$ thickness were made on gelatin precoated slides.

\section{NORTHERN BLOT ANALYSIS}

Total cellular RNA ( $20 \mu \mathrm{g})$ was isolated on a caesium chloride gradient, separated on a formaldehyde denaturing gel and transferred to Duralon (Stratagene, CA). The RNA was cross linked, prehybridized for two hours at $42^{\circ} \mathrm{C}$, and subsequently hybridised with [ ${ }^{32} \mathrm{P}$ ] labelled cDNA PDGF probes overnight. The random hexamer priming technique was used for labelling the human CDNA PDGF ( $A$ and B) and PDGF ( $\alpha$ and $\beta$ ) receptor probes. Filters were washed in $0.1 \mathrm{M}$ tri-sodium citrate plus $0 \cdot 1 \%$ sodium dodecyl sulphate at $50^{\circ} \mathrm{C}$ for 30 minutes and exposed to $x$ ray film for either 44 (fig $2 \mathrm{~A}$ ) or 24 hours (fig $2 \mathrm{~B}$ ).

IN SITU HYBRIDISATION

Around 400-500 base pair fragments from the coding regions of human cDNA PDGF clones were cloned into Bluescript plasmid (Stratagene, CA). T7 RNA polymerase generated antisense and T3 RNA polymerase generated sense $\left.{ }^{35} \mathrm{~S}\right]$ UTP (New England Nuclear) labelled riboprobes. The protocol was similar to that outlined in Current Protocols of Molecular Biology, ${ }^{4}$ with minor modifications. The slides were developed after one week and counter stained with haematoxylin and eosin.

Positive controls for each in situ experiment included cells that were shown to express the appropriate RNA by northern blot analysis (fig $2 \mathrm{~A}$ and $2 \mathrm{~B}$ ). Negative controls included RNAase pretreated sections and sense strand riboprobe (fig $3 \mathrm{~A}$ and $3 \mathrm{~B}$ ). Immunohistochemistry with specific antibodies to the two PDGF ligands and receptor subunits were undertaken on cryosections from the same block used for in situ experiments (data not shown).

\section{Results}

The most striking result was the amount of PDGF $a$-receptor mRNA expression by the glioblastoma specimen taken at OR1 (fig 2B). Many areas from OR2, analysed by in situ hybridisation, consisted of necrotic glial tissue with scattered large pleomorphic cells, expressing PDGF $a$-receptor mRNA (arrow in fig 3B). By contrast, PDGF $\beta$-receptor was undetectable in OR1 by northern analysis. The $\beta$-receptor signal was detectable by much lower compared with the PDGF $\alpha$ receptor signal (fig $2 \mathrm{~B}$ ). In situ hybridization for PDGF $\beta$-receptor on the OR2 specimen, however, showed that the number of grains localising to the large pleomorphic cells was not increased compared with the negative northern blot analysis from OR2, but was 


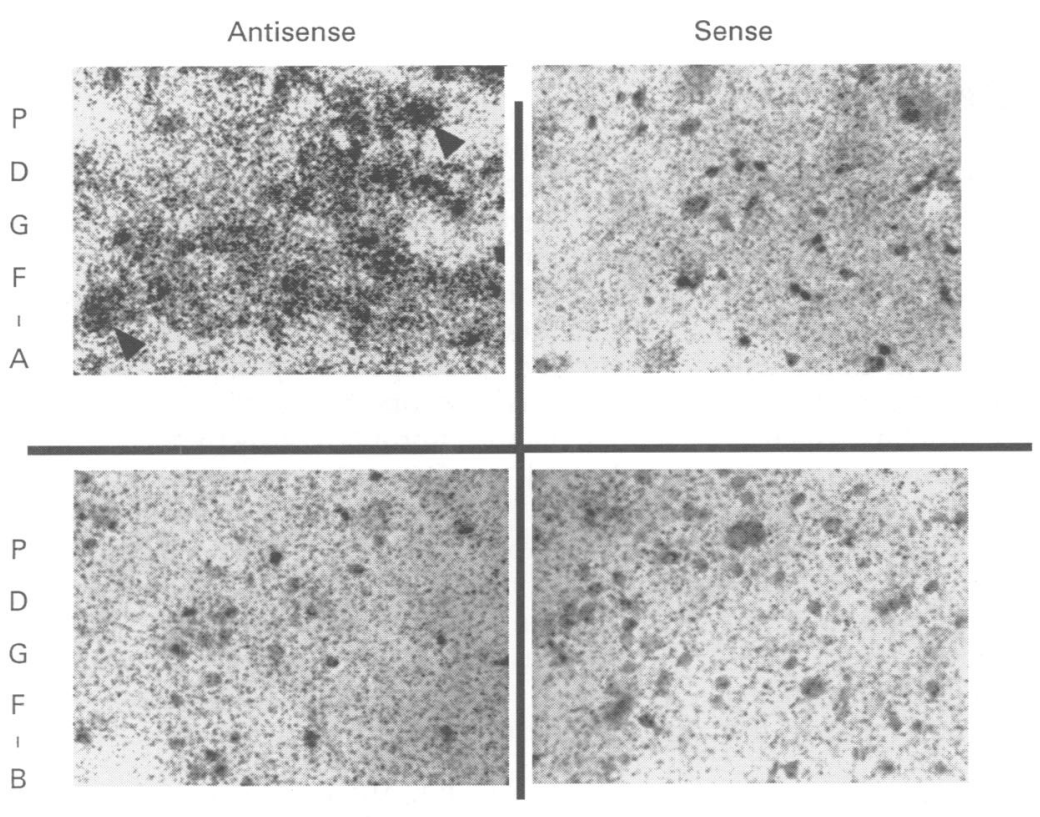

A

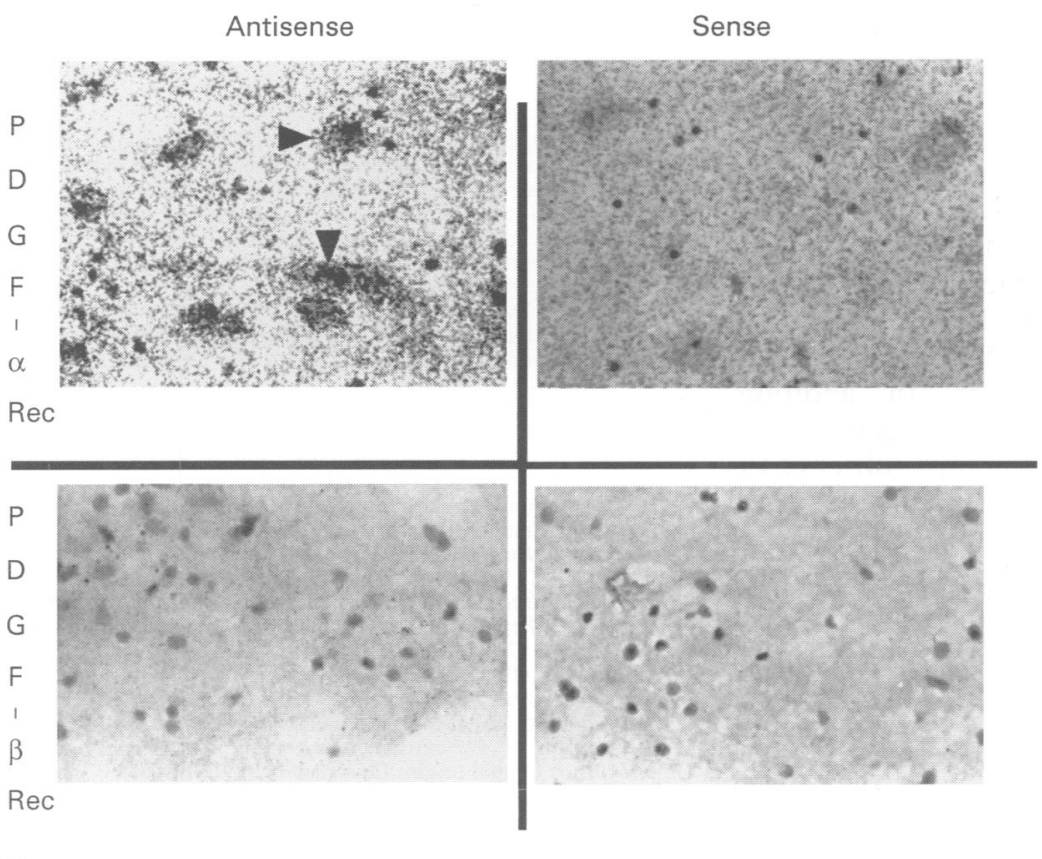

$\mathrm{B}$

Figure 3 In situ hybridisation with ${ }^{35}$ S] labelled $P D G F-A$ and $B(A)$ and $P D G F \alpha$ and $\beta$-receptor $(B)$ antisense riboprobes on cryosections of $O R 2$ glioblastoma specimen. Sense strand riboprobes served as the negative control. Increased PDGF- $A$ and $\alpha$-receptor $m R N A$ signal were localised to the large pleomorphic tumour cells (arrows), which were GFAP positive on sections taken from the same tissue block (data not shown). PDGF-B mRNA signal in these cells was also increased, but to a lesser extent when compared with the background signal from the sense strand. PDGF $\beta$-receptor $m R N A$ signal was hardly detectable.
mRNA expression was minimal, a difference that was most evident in the specimen from OR1 (fig 2A). PDGF-B mRNA was detected by in situ hybridisation in the large cells from OR2; however, the concentration of the grains were much less than for PDGF-A. Similar to the patient with Li-Fraumeni syndrome most sporadically occurring glioblastomas had increased concentrations of both PDGF-A and PDGF-B mRNA. ${ }^{3}$ Immunohistochemical analysis of adjacent tumour sections used for in situ experiments with antibodies specific to PDGF ligands and PDGF receptor subunits, showed expression similar to the data for the mRNA (data not shown).

\section{Discussion}

The identification of PDGF and its receptors in glioblastoma cell cultures and specimens from operation suggests that PDGF is an important mitogen for astrocytomas. ${ }^{35}$ In sporadic low grade astrocytomas, amounts of PDGF and PDGF receptors are lower than their malignant counterpart, suggesting that PDGF is important in the malignant progression of these tumours. This was supported by our findings that blocking PDGF stimulation of malignant human astrocytoma cell lines with PDGF dominant negative mutants, reverted their tumorogenic growth in cell cultures and nude mice. ${ }^{2}$ This study shows that the overall expressions of PDGF and PDGF receptor mRNA are similar in a patient with Li Fraumeni syndrome with a glioblastoma, and in those previously found in sporadic high grade tumours. ${ }^{3}$ Abundant PDGF-A and PDGF $a$-receptor mRNAs were expressed and localised to the large pleomorphic tumour cells, with the potential for PDGF stimulated growth. The tumour cells also expressed PDGF-B that could activate the PDGF $a$ receptor, although at lower levels than PDGF-A. In sporadic glioblastoma specimens, overexpression of PDGF $a$-receptors may reflect amplification of the $a$-receptor gene. ${ }^{6}$ In one reported case the PDGF $a-$ receptor was also rearranged, resulting in the truncation of a portion of the extracellular domain involved in binding of PDGF. ${ }^{7}$ It is not known if altered or the normal overexpressed PDGF $a$-receptors in malignant astrocytomas are active, either constitutively or through binding of the indigenously expressed PDGF.

$\mathrm{Li}$-Fraumeni syndrome was recognised in 1969 from clinical-epidemiological data of patients with sarcoma in childhood, with a high incidence of familial cancers. ${ }^{8}$ The cancers associated with the syndrome present at a young age, are often multiple, and are transmitted in an autosomal dominant fashion from one generation to the next. These characteristics of Li-Fraumeni syndrome are clearly shown in the pedigree of our patient. Presently, six types of cancers are associated with the syndrome (soft tissue sarcoma, osteosarcoma, breast cancer, acute leukaemia, adrenocortical carcinoma, and astrocytomas), all of which are pathologically similar to their 
more common sporadic counterparts. ${ }^{1}$ Astrocytomas are the third most common associated with the syndrome, often occurring before the age of 15, with the rest usually before the age of 45 . Most of these are malignant in nature and do not differ pathologically from those present in the general population.

Germ line mutations of one p53 allele were initially detected in fibroblasts from six $\mathrm{Li}$ Fraumeni families. ${ }^{1}$ Exon 5-8 was examined, as it encoded the highly conserved and DNA binding region of the p53 protein, which is probably vital for its function. Most of the p53 mutations reported from a variety of sporadically occurring cancers are clustered within this region of the p53 gene. ${ }^{9}$ Loss of activity from the remaining normal p53 allele, through a second somatic mutation or deletion, would lead to tumours in these patients at a higher rate than those not carrying the germ line defect.

Further examination of larger numbers of clinically diagnosed Li-Fraumeni families, however, showed germ line p53 mutations in only $50 \%$ of the families. ${ }^{10}$ The family in this report illustrates such a case, as no germ line p53 mutations were detected within the conserved exons $5-8$ by the polymerase chain reaction $(\mathrm{Li}$, personal communication). The underlying defect may still reside in mutations of the p53 gene, but perhaps outside the conserved regions first examined. Presently, a more extended search is being undertaken over a wider region of the $\mathrm{p} 53$ gene in this and other Li-Fraumeni families. In addition, mutations in cancer susceptible genes other than p53 may be transmitted in a subset of $\mathrm{Li}$ Fraumeni families. Amplifications of genes such as MDM2," the protein products of which bind and block the function of normal $\mathrm{p} 53$, is yet another possible genetic mechanism for a subset of Li-Fraumeni families where germ line p53 mutations cannot be identified.

Loss of one copy of the short arm of chromosome 17, near the $\mathrm{p} 53$ gene, occurs in $30 \%$ to $50 \%$ of sporadic astrocytomas. ${ }^{12}$ Mutations in the p53 gene between exons 5-8 (conserved region of p53), are present in about one third of all astrocytomas, and two thirds of those tumours with loss of one p53 allele. Although some reports suggest that p53 deletions are only present in higher grade astrocytomas, recent reports show p53 mutations to be a relatively early event. Sidransky et $a l^{13}$ reported that a small subpopulation of low grade astrocytoma cells had p53 mutations. The growth advantage provided from inacti- vation of p53 resulted in these cells becoming the dominant cell type as the tumour progressed to a higher grade.

The molecular pathogenesis of sporadic astrocytomas still remains unclear. Previous results suggest that PDGF provides mitogenic stimulation for malignant astrocytomas, a pathogenetic mechanism that is possibly common to development of these tumours in a genetically predisposed patient. Whether other growth factors or receptors (epidermal growth factor, fibroblast growth factor, etc) implicated in the pathogenesis of sporadic malignant astrocytomas are also found in these tumours associated with $\mathrm{Li}$-Fraumeni syndrome needs to be studied. Examination of rare familial cancer syndromes should allow greater insights into their more common sporadic counterparts, as their molecular pathogeneses are likely to be similar.

We thank Fredrick P Li MD and Margaret G Dreyfus BA from the Division of Cancer Epidemiology and Control, from the Division of Cancer Epidemiology

A Guha was supported by a Clinical Scientist Award from A Guha was supported by a Clinical Scid
the Medical Research Council of Canada.

1 Malkin D, Li FP, Strong LC, et al. Germ line p53 mutations in a familial syndrome of breast cancer, sarcomas, tions in a familial syndrome of breast cancer, sarc
and other neoplasms. Science 1990;250:1233-8.

2 Shamah S, Stiles C, Guha A. Dominant-negative mutants of platelet-derived growth factor (PDGF) revert the transformed phenotype of human astrocytoma cells. Mole Cell Biol 1993;13:7203-12.

3 Guha A, Dashner K, McL. Black P, Wagner J, Stiles C. Expression of PDGF and PDGF-receptors in human astrocytoma operative specimens. Int $\mathcal{f}$ Cancer 1995;60: 168-73.

4 Ausbel F, Brent R, Kingston R, Moore D, Seidman J, Struhl K, eds. Current protocols in molecular biology: in situ hybridization. Vol 2. New York: Wiley, 1989: chapter 14.

5 Nistér M, Claesson-Welsh L, Eriksson A, Heldin C-H, Westermark B. Differential expression of platelet derived growth factor receptors in human malignant glioma cell growth factor receptors in human malign
lines. $\mathcal{F}$ Biol Chem 1991;266:16755-63.

6 Fleming T, Saxena A, Clark W, et al. Amplification and/or overexpression of platelet derived growth factor receptors and epidermal growth factor receptor in human glial tumors. Cancer Res 1992;52:4550-3.

7 Kumabe T, Sohma Y, Kayama T, Yoshimoto T, Yamamoto $T$. Amplification of A-platelet-derived growth factor receptor gene lacking an exon coding for a portion of the extracellular region in a primary brain tumor of glial origin. Oncogene 1992;7:627-33.

$8 \mathrm{Li} \mathrm{F}$, Fraumeni J Jr. Soft tissue sarcomas, breast cancer, and other neoplasms: a familial syndrome? Ann Intern Med 1969;71:747-52.

9 Hollstein M, Sidransky D, Vogelstein B, Harris CC. p53 Mutations in human cancers. Science 1991;253:49-53.

10 Santibanez-Koref M, Birch J, Hartley A, et al. p53 Germline mutations in Li-Fraumeni syndrome. Lancet 1991;338:1490-1.

11 Oliner J, Kinzler K, Meltzer P, George D, Vogelstein B. Amplification of a gene encoding a p53 associated protein in human sarcoma. Nature 1992;358:80-86.

12 Frankel $R$, Bayona W, Koslow M, Newcomb E. p53 Mutations in human malignant gliomas: comparison of loss of heterozygosity with mutation frequency. Cancer Res 1992;52:1427-33.

13 Sidransky D, Mikkelsen T, Schwechheimer K, Rosenblum $M$, Cavanee W, Vogelstein B. Clonal expansion of $p 53$ mutant cells is associated with brain tumour progression. Nature 1992;355:846-7. 